\title{
Occurrence of Measles among Children Admitted in Tertiary Care Hospital
}

\author{
MOHAMMED RIZWANULAHSAN ${ }^{1}$, ABDULLAH AL MAMUN² ${ }^{2}$, HOSSAIN SHAHID KAMRUL ALAM ${ }^{3}$, \\ PROBIR KUMAR SARKER ${ }^{4}$, SABRINA MAKBUL ${ }^{5}$, ROWSHAN KABIR ${ }^{6}$, \\ ABM MAHFUJ HASSAN AL MAMUN ${ }^{7}$
}

\begin{abstract}
Background: Despite widespread vaccination against measles in Bangladesh outbreak continue to occur. The Objective of the study was to see the demographic characteristics of patients who had the diagnosis of measles at Dhaka Shishu (Children) Hospital and occurrence of measles from the time of vaccination.

Methodology: This retrospective study was conducted on patient who were diagnosed as measles at Dhaka Shishu (Children) Hospital between $1^{\text {st }}$ January 2017 to $30^{\text {th }}$ April 2017. The diagnosis of measles based on clinical features and laboratory confirmation. Demographic characteristics of patients and occurrence of measles from the time of vaccination was recorded. Data were analyzed by using SPSS version 21.

Results: Total 13864 patients were admitted during the study period and among them 23 were confirmed as measles. Rate of measles was found as $0.16 \%$ among hospitalized children with male predominance. Among the measles patients age of onset was $<9$ months in 2(8.7\%), 9-12 months in 8(34.8\%), 13-14 months in 11(47.8\%) and 15 months and above in 2(8.6\%) cases. Among 23 measles patients vaccine was not given in 2(8.7\%) patients, one dose of vaccine was given in $19(82.60 \%)$ patients and two doses of vaccine were given in $2(8.7 \%)$ patients. Most of the measles patients came after one vaccination which means after first dose. Though measles incidence significantly reduced after the successful introduction of measles vaccination in EPI, still measles are prevalent in our country and occurs before completion of two doses of vaccine.
\end{abstract}

Conclusion: Majority of measles cases developed before completion of two doses of vaccine though the successful measles vaccination are going on.

Key words: Measles, Vaccination.

1. Assistant professor and Resident Physician, Dhaka Shishu (Children) Hospital.

2. Associate Professor, Department of Cardiology, Dhaka Shishu (Children) Hospital.

3. Associate Professor, Department of Adolescent Pediatrics, Dhaka Shishu (Children) Hospital.

4. Associate Professor, Department of Rheumatology, Dhaka Shishu (Children) Hospital.

5. Sabrina Makbul, Senior Nutritionist, Dhaka Shishu (Children) Hospital.

6. INFS, Dhaka University

7. RMO, Department of Adolescent Pediatrics, Dhaka Shishu (Children) Hospital.

Correspondence to: Dr. Mohammed Rizwanul Ahsan, Assistant Professor and Resident physician, Department of Adolescent Pediatrics, Dhaka Shishu (Children) Hospital. Email: rizwanul.ahsan@gmail.com. Mob: +8801711465405

Received: 21 September $2017 \quad$ Accepted: 13 March 2018

\section{Introduction}

In large parts of the world vaccination has successfully eliminated measles as a significant causes of morbidity and mortality. ${ }^{1}$ However, it is one of the leading causes of child mortality and in 2011, contributed 158000 deaths globally. ${ }^{2}$ Measles virus is highly infections and is transmitted by aerosols generated during coughing or by direct contact with contaminated respiratory secretion. After an incubation time of almost two weeks, disease starts with a prodromal phase of fever cough and coryza. A few days later a generalized maculopapular skin rash appears, often in combination with conjuctivities. ${ }^{3}$ Measles is associated with a transient but profound 
immunosuppression, resulting in an increased susceptibility to opportunistic infections. This often leads to complications like pneumonia, diarrhoea and otitis media which are the most important determinants of measles morbidity and mortality. 3,4 Paradoxically, the disease is also associated with the induction of strong measles virus specific humoral and cellular immune response, resulting in life-long immunity. ${ }^{5}$

Before the introduction of the measles vaccine, the measles virus caused millions of deaths worldwide. ${ }^{6}$ Measles vaccination is the only hope to reduce incidence and mortality of this disease. WHO recommends two doses of vaccination with flexibility of schedule as $15 \%$ of vaccinated children fail to develop immunity from first vaccination dose. However, routine vaccination in many countries have caused measles to become relatively uncommon. In Bangladesh, with the successful implementation of the Expanded Programme on Immunization, measles incidence declined. During 2000-2016, incidence of measles cases decreased $84 \%$, from 40.0 to 6.0 per million. ${ }^{7}$

Measles is relatively uncommon among infants of developed countries due to the protection offered by maternal antibodies. In developing countries, where there is a high degree of wild-type measles transmission, infants and children are at greater risk of being exposed shortly after maternal antibodies have waned. This often occurs as early as at 5-6 months of age. $^{8}$

Measles at an early age is associated with serious complications and an increased risk of mortality, necessitating vaccinations as early as possible. In developed countries live attenuated vaccines are safe, immunogenic and effective. However, due to the early loss of maternal antibodies in infants in developing countries, it has been recommended that measles vaccine should be administered from 9 months of age. ${ }^{9}$ This strategy, however, leaves a window of high risk between 4 and 9 months of age.

In this report, we presented demographic characteristics of patients who had the diagnosis of measles at Dhaka Shishu (Children) Hospital and occurrence of measles from the time of vaccination.

\section{Materials and Methods}

This retrospective study was conducted on patient who was diagnosed as measles at Dhaka Shishu
(Children) Hospital, the largest tertiary care pediatric hospital in Bangladesh, between $1^{\text {st }}$ January 2017 to $30^{\text {th }}$ April 2017. The study was approved by Ethical Committee of Dhaka Shishu (Children) Hospital. The disease was diagnosed with clinical suspicion and laboratory confirmation, standard isolation airborne precautions were applied for every patient who was suspected measles until the result of ELISA. The diagnosis of measles was confirmed by measles specific immunoglobulin $\mathrm{M}(\lg M)$ antibody positivity in serum sample and was included in the study. After selected the cases reporting done sample has collected and sent to the NPB and report has collected after seven days. This study confirmed measles serologically, thus avoiding the potential bias in vaccine effectiveness from non-specific diagnosis. Age, gender, vaccination status, clinical and laboratory findings were recorded. All relevant data was extracted from chart and electronic records from the hospital. Data were analyzed by using SPSS version 21.

\section{Results}

Total 13864 patients were admitted during the study period and among them 23 were confirmed as measles. Rate of measles was found as $0.16 \%$ among hospitalized children. There were $17(73.90 \%)$ male patients and $6(26.10 \%)$ female patients with a male female ratio of 2.8:1 (Fig.-1).

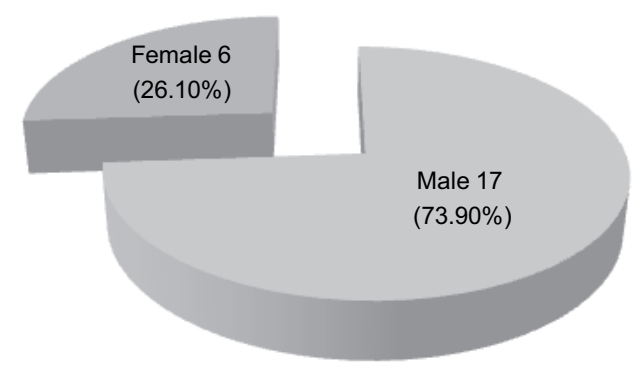

Fig.-1: Pie diagram of respondents according to gender ( $n=23)$

Among 23 measles patients vaccine was not given in $2(8.7 \%)$ patients, one dose of vaccine was given in $19(82.60 \%)$ patients and two doses of vaccine were given in $2(8.7 \%)$ patients. Most of the measles patients came after one vaccination which means after first dose (Fig.-2). 
Two doses of

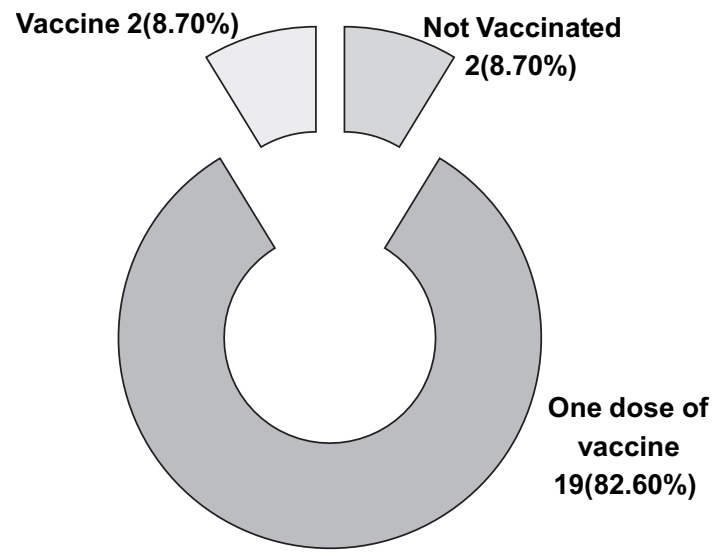

Fig.-2: Vaccination status of the study population $(n=23)$

Among the measles patients age of onset was $<9$ months in 2(8.7\%), 9-12 months in 8(34.8\%), 13-14 months in $11(47.8 \%)$ and 15 months and above in $2(8.6 \%)$ cases (Fig.-3).

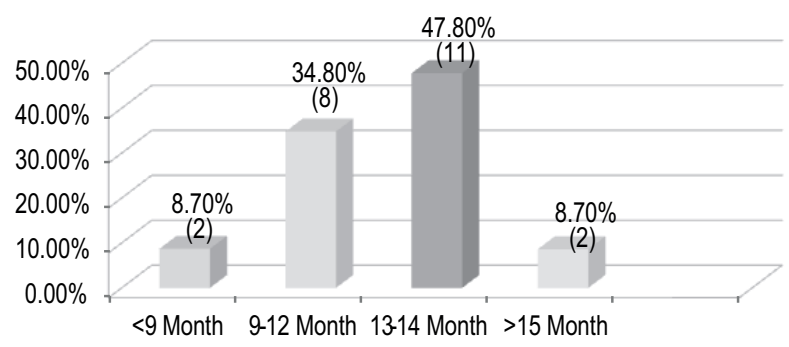

Fig.-3: Bar diagram represents the respondents according to age of onset of measles $(n=23)$

\section{Discussion}

In this study male preponderance is observed. Same results have been reported by Khan et al $^{10}$ and Mood et $\mathrm{al}^{11}$

Among the measles patients age of onset was $<9$ months in 2(8.7\%), 9-12 months in 8(34.8\%), 13-14 months in $11(47.8 \%)$ and 15 months and above in $2(8.6 \%)$ cases. Akramuzzaman et al in Bangladesh found $23 \%$ of measles cases before 9 months of age and $19 \%$ between $9-11$ months of hospitalized children admitted with measles. ${ }^{12}$

In Bangladesh the timing of the first dose of measles vaccine at 9 months of age to minimize interference from maternal antibody and to provide protection for the maximum number of infants. Among 23 measles patients vaccine was not given in $2(8.7 \%)$ patients, one dose of vaccine was given in $19(82.60 \%)$ patients and two doses of vaccine were given in $2(8.7 \%)$ patients. Most of the measles patients came after one vaccination which means after first dose. WHO recommends delayed measles vaccination until maternal antibody has waned. Research in the USA has shown that cell mediated responses to measles vaccine given to children at 6 months of age were similar to those in children vaccinated at 9 or 12 months of age but antibody responses were diminished by maternal antibody. However 6 months after a boost at 12 months of age protective levels of antibody were achieved in $86 \%$ of the youngest children while T-cell proliferative responses changed little in any of the age groups. ${ }^{13}$ Vaccine effectiveness of an early two dose schedule during a large measles epidemic in Florida was $99 \% .{ }^{14}$ An additional early measles vaccine given to children at 4 months of age induced a predominant CD4 T-cell response at 9 months and rapid development of high antibody concentrations after booster doses. ${ }^{15}$ In low income countries, maternal antibody levels against measles may be low and severe outbreaks of measles can occur in infants before the recommended age of vaccination at 9 months. ${ }^{16}$ However, early measles vaccine may improve child survival by reducing mortality. ${ }^{17}$ Early two dose schedules of measles vaccine given at 6 and 9 months of age were recommended by WHO to control outbreaks and for use in countries with high attack rates of measles in infancy. Now WHO recommends such schedules in areas with a high incidence of HIV and measles. ${ }^{18} \mathrm{An}$ early two dose schedule would fit well and protects the very young and enhances child survival. ${ }^{16,17}$

The current measles vaccination policy was based on children born to naturally infected mothers. Such children may not lose protection from maternal antibodies until around 7 to 9 months of age. ${ }^{19}$ The situation has changed since the 1980s. Most low income countries have had measles vaccination programmes for the past 30-35 years. As a consequence many mothers have been immunized and have not had natural measles infection. A new group of children born to immunized mothers therefore exists who may lose protection early. ${ }^{16}$ This situation should lead to reconsideration of the policy for measles vaccination. An early two dose measles vaccination strategy could strengthen the current efforts to control measles and reduce measles mortality. 


\section{Conclusion:}

Majority of measles cases developed before completion of two doses of vaccine though the successful measles vaccination are going on.

\section{References:}

1. De Quardos CA, Izurieta H, Carrasco P, Tambini $G$. progress towards measles eradication in the region of the American. J Infect Dis.2003;187 (Suppl 1):S102-S110.

2. World Health Organization (WHO) Measles fact sheet No 286. Genava:WHO; Updated 2013 Feb 20; Cited 2013 Nov 14. Available from: http:// www.who.int/mediacentre/factsheets/fs286/en/ index.html.

3. World Health Organization. Progress in reducing global measles deaths: 1999-2004. WklyEpidemiol Rec. 2006;81:90-94.

4. Griffin DE. Measles virus. In: Knipe DM, HowleyPM,eds. Fields Virology. $5^{\text {th }}$ ed. Philadelphia, PA: Lippincott Williams \& Wilkins;2007:1551-1585.

5. Rima BK, Duperx WP. Morbilliviruses and human disease. J Pathol.2006;208:199-214.

6. Anonymous. Measles immunisation: time to close the gap. Lancet Infect Dis 2016;16:1.

7. Khanal S, Bohara R, Chacko S, Sharifuzzaman M, Shamsuzzaman M, Goodson JL. Progress Toward Measles Elimination - Bangladesh, 20002016. Centers for disease control and Prevention. Available at: https://www.cdc.gov/mmwr/ volumes/66/wr/mm6628a3.htm

8. Garenne M, Aaby P. Pattern of exposure and mea- sles mortality in Senegal.Journal of Infeczious Diseases.1990; 161: 1088-94.

9. Black FL, Berman LL, Borgono JM. Geographic variation in infant loss of maternal antibody and in prevalence of rubella antibody. Am J Epidemiol 1986;124:422-52.

10. Khan M, Khan KMA, Ahmed A. Audit of measles cases in a tertiary care hospital; Pak Paediatr J 2013:37(3):143-8.

11. Mood BS, Naini RN, Salehi M, Kouhpayeh HR, Azad TM, Poor TN. Immunity against measles among vaccinated school going children in Zahedan, Southeast of Iran. Indian J Med Microbiol 2005; 23(4): 274-75.

12. Akramuzzaman SM, Cutts FT, Hossain MJ, Wahedi OK, Nahar N, Islam D. Measles vaccine effectiveness and risk factors for measles in Dhaka, Bangladesh. Bulletin of the World Health Organization 2002; 80:776-82.

13. Gans H.A., Yasukawa L.L., Alderson A., Rinki M., DeHovitz R., Beeler J. Humoral and cellmediated immune responses to an early 2-dose measles vaccination regimen in the United States. J Infect Dis. 2004;190:83-90.

14. Hutchins SS, Dezayas A, Le Blond K, Heath J, Bellini W, Audet S. Evaluation of an early twodose measles vaccination schedule. Am J Epidemiol. 2001;154:1064-1071.

15. Njie-Jobe J, Nyamweya S, Miles DJC, van der Sande M, Zaman S, Touray E. Immunological impact of an additional early measles vaccine in Gambian children: Responses to a boost at 3 years. Vaccine. 2012 Mar 28; 30(15): 2543-50.

16. Martins CL, Garly ML, Bale C, Rodrigues A, Ravn $\mathrm{H}$, Whittle HC. Protective efficacy of standard Edmonston-Zagreb measles vaccination in infants aged 4.5 months: interim analysis of a randomised clinical trial. BMJ. 2008;337:a661.

17. Aaby $\mathrm{P}$, Martins $\mathrm{CL}$, Ravn $\mathrm{H}$, Rodrigues $\mathrm{A}$, Whittle HC, Benn CS. Is early measles vaccination better than later measles vaccination? Transactions of The Royal Society of Tropical Medicine and Hygiene. 2015;109: 1628.

18. WHO position on measles vaccines. Vaccine. 2009;27:7219-7221. Available at https:/ /www.ncbi.nlm.nih.gov/pmc/articles/ PMC3401374.

19. Jensen TG, Whittle H, Mordhorst $\mathrm{CH}$, Pedersen $I R$, Thårup $\mathrm{J}$, Poulsen $\mathrm{A}$, et al. Trials of Edmonston-Zagreb measles vaccine in GuineaBissau: serological responses following vaccination with Edmonston-Zagreb strain at 48 months versus vaccination with Schwarz strain at 9-12 months of age. Vaccine1994;12:1026-31. 